\title{
Measurement Eccentricity the Moon's Orbit with Image Analysis Technique by Using Tracker Software
}

\author{
Ricka Tanzilla ${ }^{1}$, Ishafit ${ }^{2}$ and Yudhiakto Pramudya ${ }^{3}$ \\ I, 2, ${ }^{3}$ Physics Education,Universitas Ahmad Dahlan, Indonesia \\ J1. Prof. Dr. Soepomo SH, Janturan Warungboto Yogyakarta \\ E-mail: tanzilla.ricka@gmail.com
}

\begin{abstract}
One of the interesting physical phenomenon to be studied isabout eccentricity of the Moon's orbit on the movement of the Earth and the Moon as explained in Kepler's Law. In order to explain the phenomenon,several research has been done to determine themeasurement accuracy of Moondiskdiameter using Trackers and to know the result of the eccentricity value in the application of Kepler's Law. This research can produce eccentricity values accordance with the application of Kepler'sLaw. The research method used is ImageAnalysis Techniqueassisted with Tracker. The result of this research shows that the eccentricityvalue obtained is $0.07 \pm 0.0 \mathrm{I}$. This is in accordance withKepler's first Law which stated that orbits of each planet andsatellite is an ellipse and has an eccentricity value of $0<\mathrm{e}<\mathrm{I}$. The value of eccentricity obtained has accuracy of $22 \%$, if it compared with the actual value. Whereas, if it compared with the value of ephemeris then the value of accuracy is $8 \%$.
\end{abstract}

Keywords: Kepler's Law, Eccentricity, Moon, Tracker

\section{Introduction}

Physics is the study of nature and symptom of objects in the universe with various physical phenomenon such as; the movement of celestial objects, changing process of moon phases, etc. [I]. Physics consists of various branches of science. An interesting branch of science to study is astrophysics. The aim of astrophysics is to find out the physical nature of objects in the universe [2]. Physical phenomenon related to the universe can be studied through physics. Even those that are far from the reach of the eyes. As the basis of astrophysical science, one thing to know is the movement of the Planet. Planetary movements can be explained through Kepler's Law. Kepler's Law was initiated by a classical physics scientist, Johannes Kepler [3].Kepler explains the movement pattern of planets surrounding the Sun and other celestial objects. FromKepler's observations it turns out that it agrees with what Copernicus has stated is that planets do not surround the Earth but surround the Sun [4].Kepler's and Copernicus' laws have difference. The difference is, according to Kepler the orbit is in the form of an ellipse, while according to Copernicus the orbit is in the form of a circle [5].JohannesKepler and Tyco Brahe made more precise measurements so that the results obtained were more accurate. Therefore the results of the observations and analysis were concluded in the form of 3 empirical laws regarding the movement of the Planet calledKepler's Law [6].What is expressed "Empirically" shows that these laws are not based on deep theory, but accurately describe the observed motion of the planet. Kepler's first Law and Kepler's second Lawwere first published in 1609 and Kepler'sthird Law was published in 1618 [7]. Kepler's first Law states all planets surround the Sun in an elliptical orbits, having the Sun as one of the foci.Kepler's second law states a radius vector joining any planet to the Sun sweeps out equal areas in equal lengths of time. Kepler's third Law, the squares of the sidereal periods (of revolution) of the planets are directly proportional to the cubes of their mean distances from the Sun.Kepler uses Planet to prove his physical law [3].

Kepler's law that will be studied in this study is Kepler's first Law. Most physics books are still stated that all planets surround the Sun in an elliptical orbits, having the Sun as one of the foci. Whereas, what has happened at this time there are many studies showing that not only planets surround the Sun in elliptical orbits. But this also happens with the satellites that surround the Planet. For example, the Moon surrounds the Earth in an elliptical orbit. [3].

There are a lot of methods that show that the Moon's orbit is an ellipse. Research by E. Jay Sarton under the title Measuring the moon's orbit by using projector screen projection to measure the Moon's diameter so that it 
obtained the results of the Moon's orbit eccentricity that is 0.0580 [8].

Seeing from the lack of the previous research, this study will design eccentricity measurements of the Moon's orbit by using Tracker so that it will be easier to process the image produced from the Moon. Moon image is then calculated the Moondisk diameter image in pixel and then converted into the moon angle diameter. This experiment was conducted because it knew the accuracy of the diameter measurement of the Moon disk using Tracker and knowing the results of eccentricity values in the effort to implementKepler's Law.

\section{Literature Review}

Research on the application of Moon diameter for measuring the eccentricity of the Moon's orbit has been be inspected by Banjamin Oostra in an experiment entitled Measuring the Moon's orbit using a hand-held camera the method used by taking pictures consistently and measuring the visible diameter in the picture. In this study the measurement of the diameter of the Moon's appearance using the Iris program. In this study the measurement of the diameter of the Moon's appearance using the Iris program. After obtaining the diameter value, it appears that the next step is analyzed so that the eccentricity value of the Moon's orbit is 0.047 . Eccentricity of the Moon's orbit is obtained from elliptical geometry analysis. The agreed value is that the eccentricity value of the Moon's orbit is 0.0549 [9]. Although the results are inaccurate, this research is able to indicate a simple method to measure the eccentricity of the Moon's orbit [IO].

Subsequent research was carried out by Jorge I. Zuluaga et al. [II] entitled Thesimplest method to measure the geocentric lunar distance: a case of citizen science. This study describes the results of the Moon's geocentric distance measurement which aims to explain simple methods with the results of the prescription. This measurement uses the Moon's geocentric distance counting method only with images and measurements of the precision of the moon's visible diameter measurements. Measuring the diameter of the Moon's appearance using the Python Matplotlib application. So that the geocentric measurements of the Moon distance are obtained by D / RE $=60.5$ I [I I].

Subsequent research was carried out by Benjamin Oostra [IO] with the title Introducing the Moon's Orbital Eccentricity. This research provides a way to introduce the eccentricity of the Moon's orbit in the introductory class of Astronomy. The method used is to exercise direct observation with students to determine the eccentricity of the Moon's orbit. Moon images taken when Moon is in the position of Perigee and Apogee by using a 12 megapixel camera phone with 12 times the optical zoom shows variations in size due to different distances. The eccentricity value obtained is 0.043 [I2].

The next research was carried out by Kevin Krisciunas with the title Determining the eccentririty of the Moon's orbit without a Telescope. This study was able to measure the eccentricity of the Moon's orbit with a value of $e=0.039 \pm 0.006$. This research uses a piece of cardboard with a small hole inside which slides up and down with a ruler that allows measuring eccentricity [13].

Subsequent research was carried out by Tanzilla et al. [I4] with the title Measurement of the Visible Moon Diameter on the Position of Perigee and Apogee. This study uses GIMP Software to help calculate the Moon's Visible Diameter. The percentage comparison value when lunar Apogee and Perigee is $15.77 \%$ [I4].

\section{a. Kepler's Law}

From the study of observational data on the position of the planets collected by Johannes Kepler with Tycho Brahe's formulating three laws of planetary motion which are always associated with their names. The three laws are: I) Planet move around the sun in ellipses, with the Sun at one focus

2) The line connection the Sun to a planet sweeps equal areas in equal time.

3) The square of the orbital period of a planet is proportional to the cup (3rd power) of the mean distance from the Sun

The first law states that the orbit of Mars and implicitly all other planets is an ellipse with the Sun in one focus. An ellipse is a form obtained by slicing a cone with a plane at an angle to its base. This is a symmetrical and well-defined form with two focus and properties. The length of a line or string from one focus to any point in the ellipse and then to the other focus is constant [I5].

The second law states that the speed of a planet varies so that the line that connects to the Sun sweeps out the same area at the same time. So, when the planet is closest to the Sun (perihelion) it moves most rapidly and if it is the furthest distance (aphelion), it moves the slowest. Although Kepler takes nine years to find and publish its third planetary movement law. This law concerns the relationship between the period of the planet and their distance from the Sun. The first is known precisely because of the large number of revolutions that have been observed since ancient times, but the distance is very unknown so there is no basis for determining the period varies with distance [16].

\section{b. Moon}

The moon is one of the natural satellites of the Earth [I7]. The distance of the Moon to Earth is only around 
Tanzilla et al.

$384,400 \mathrm{Km}$ [18], so that the Moon's radiance to Earth only takes about one second less (the speed of light is $300,000 \mathrm{~km} /$ second). The moon is the five largest satellites in the solar system and only on the Moon is the only natural satellite that has ever been footed by humans. The diameter of the Moon is much smaller than the Earth, which is only $3,474 \mathrm{~km}$ while the Earth is 12,742 $\mathrm{km}$ [19]. The moon has a surface that is never illuminated by the Sun. This is due to the axial minimum slope of the Moon, and has an impact on the crater area and other topography near the Kurub. The moon remains protected from the Sun directly throughout the year [20].

\section{c. Ellipse Geometry}

Kepler's first law states that the planets revolve the sun in the form of elliptical orbits with the sun at one focal point of the ellipse. Ellipse geometry will provide an explanation of Kepler's first law shown infigure I[2].

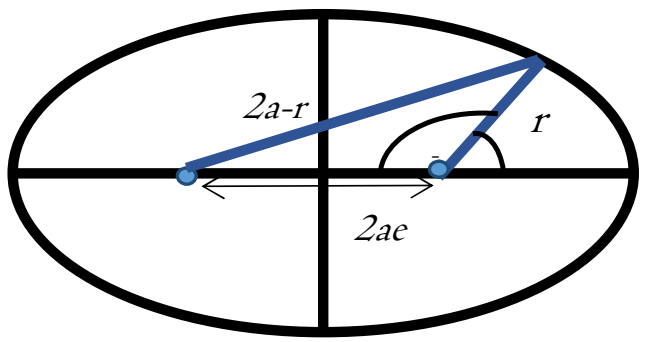

Figure I. Ellipse geometry in polar coordinates

Subsequently, by remembering $\cos (\pi-\theta)=-\cos (\theta)$, as a result,

$$
\begin{aligned}
& 4 a^{2}-4 a r+r^{2}=4 a^{2} e^{2}+r^{2}+4 r a e \cos (\theta) \\
& 4 a^{2}-4 a r=4 a^{2} e^{2}+4 r a e \cos (\theta) \\
& a^{2}-r=a^{2} e^{2}+r e \cos (\theta) \\
& a\left(1-e^{2}\right)=r(1+e \cos (\theta) \\
& r=\frac{a\left(1-e^{2}\right)}{1+e \cos (\theta)}
\end{aligned}
$$

or

$$
r=\frac{p}{1+e \cos (\theta)}
$$

with

$$
p=a\left(1-e^{2}\right)
$$

This is an ellipse in the polar coordinates, $p$ referred as ellipse parameters.

$$
\begin{aligned}
\text { If } \theta & =0^{0} \\
r_{p} & =a(1-e)
\end{aligned}
$$

Indonesian Review of Physics, Volume I, Number I, 2018 Measurement Eccentricity the Moon's Orbit with Image Analysis...

It is termed as perifocal distance. If $\theta=180^{\circ}$ thus,

$$
r_{a}=a(1+e)
$$

This is called as apfocus distance.

With:

$a=$ one half of the major axis (semimajor axis)

$r=$ radius

$a e=$ focus distance to ellipse foci

The site of points with the distance number to the fixedfocus pointis called as ellipse.

In the satellite (moon) movement around the earth, the distance is referred as apogee and perigee.

The orbit of celestial body in Kepler orbit is not only ellipse, but in general, the orbit has a conic section form like in Figure 2 which has an eccentricity value. Eccentricity is the oval sizeofellipse[2I].

The eccentricity values can be described as follows:

a. If the orbit has 0 eccentricity $(e=0)$, it called as circularorbit

b. If the orbit hasthe eccentricity between 0 and I $(0<e<\mathrm{I})$, it called as elliptic orbit

c. If the orbit has I eccentricity $(e=\mathrm{I})$, it called as parabolic orbit

d. If the orbit has the eccentricity greater than I $(e>I)$, it called as hyperbola

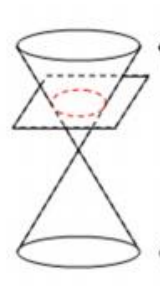

Lingkaran

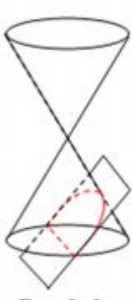

Parabola

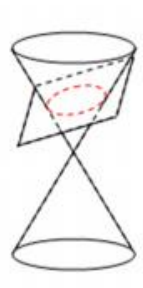

Elips

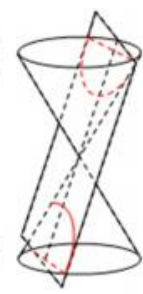

Hiperbola
Figure 2. Conic section[I5]

\section{Research/ Experiment Method}

In this research, the data collection was conducted at the $4^{\text {th }}$ campus of Universitas Ahmad Dahlan, Ring Road Selatan street, Tamanan, Banguntapan, Bantul Yogyakarta at 7०50”Southern Latitude and I 10²2”East Longitude. The subject in this study is the depiction of the Moon. The collection of Moon image is done by using the Prime Focus Technique. The data that have been taken are processed by Image analysis technique in the Tracker. Research instrument can be seen in Figure 3. 


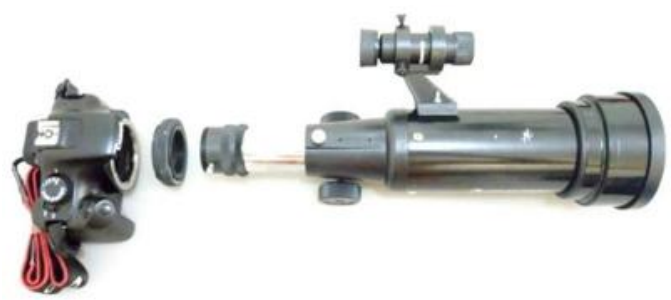

Figure 3. Research instrument

The research instrument (Figure 3) was used to capture the full Moon depiction.The Full Moon Depictionwhich was obtained is process to get the value of Moon disk diameter. Moon disk diameteris used to find the eccentricity value of Moon orbit.

\section{a. Determine the Moon diameter by using Tracker}

I) Insert the Moon depiction on Tracker and begin the push by using line profilein the Full Moon depiction.

2) In everyline profileon Trcaker, take the value intable $x$ and then processed in excel in figure 4.

3) The sky arena in the Moon depiction has a value lower than the Moon disk. There is transition from the low value to high value that occurred in the edge of the Moon disk. Transition value is used as threshold valueto determine the edge of the Moon disk. To make it easier to get the threshold value, then use themoving average. Luminosity value in every area pixcel near the Moon disk and sky is processed to obtain the difference value between adjacent pixcel. The difference in value is up and down randomly, then smoothed with a moving average. There is a significant change. This value is used as the threshold value for the determination of the edge of Moon disk. After getting the transition value, maka dapat ditetukan titik tepi piringan Bulan. This point is referred as $x r$ and $x 2$. The $x$ value has pixcel unit.

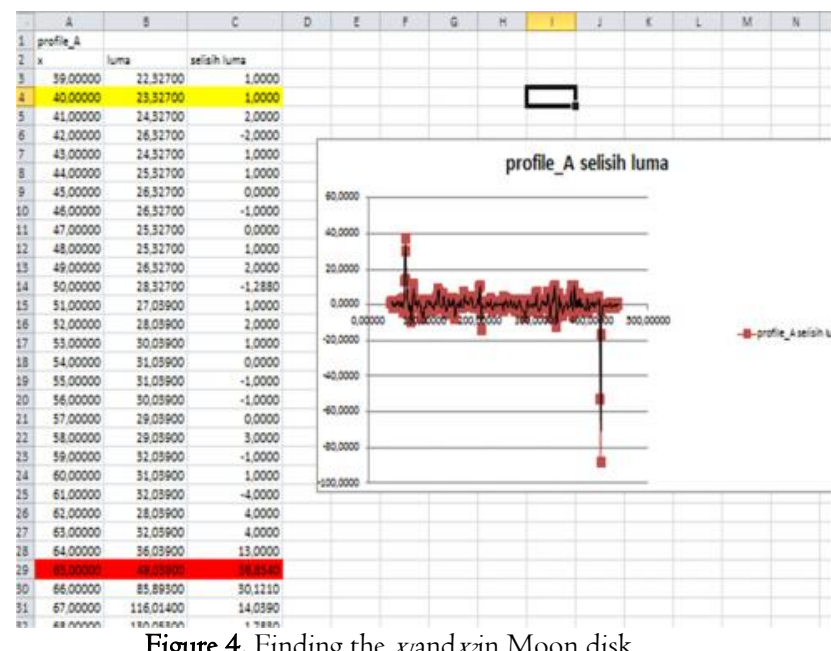

Figure 4. Finding the $x r a n d x z$ in Moon disk

4) After getting the $x I$ dan $x z$ value, we can calculate the value of the Moon disk diameter which is displayed
Indonesian Review of Physics, Volume I, Number I, 2018 Measurement Eccentricity the Moon's Orbit with Image Analysis...

in each line of profile by counting the difference between $x_{\text {rand }} x_{2}(\Delta x)$.

To determine the diameter of Moon disk, then graphs are made for each line profile and difference valueof $x(x)$,

To get the value of Moon disk diameter more accurately, we can use the equation (6)

$$
\begin{aligned}
& r_{n}=\sqrt{\left(y_{n}-y_{0}\right)^{2}+\left(\frac{1}{2} \Delta x_{n}\right)^{2}}, \\
& D=2 r_{n}
\end{aligned}
$$

tris radius value of Moon disk in each line profile, ynis the yvalue that obtained in every line profile, while yois the walue in the line profile which is considered as diameter focal point of Moon disk. xnis the difference valueof xon each line profile. Hence,the diameter of Moon disk is obtained by averaging the $i_{n}$ value then multiplied by 2 following the equation of a Circle.

\section{b. Calculating the eccentricity value}

In order to obtain the value of angular diameter $(\theta)$ of depiction, then look for nominal angular resolution of the camera (NAR) with equation (8) in rad/pixcel. After the $N A R$ value is obtained, multiply it by the diameter of the Moon disc (equation 7), so the angular diameter value is obtained.

$N A R=\left(\frac{\text { weidht_sensor_dimension }}{\text { width_image_dimension }}\right) \div$ focal_length_telescope

$\theta$ is angular diameter. The Angular diameteris affected by the distance of Earth and the Moon as shown in Figure 5.If the distance of Moon is closer from Earth, thus theangular diameteris bigger. It is called asangular diameterperigee $(\theta p)$.Whereas, if the distance of the Moon is further from Earth, the angular diameter is smaller, it is called asangular diameterapoge $(\theta a)$. The relation betweenangular diameterand distance can be described by equation $(9)$.

$$
\begin{array}{ll}
\theta=\frac{\text { diameter tampak Bulan }}{\text { jarak }} \\
\theta=\frac{D}{r} & \text { Or }
\end{array}
$$

Then, the eccentricity value that obtained is

$$
e=\frac{\theta_{p}-\theta_{a}}{\theta_{a}+\theta_{p}}
$$




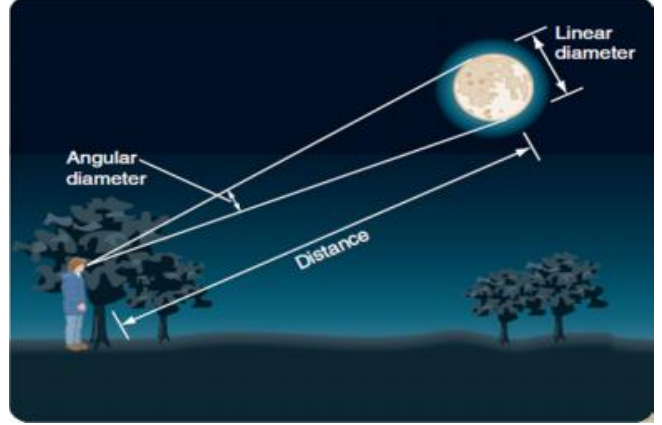

Figure 5. The angular diameter of an object(source: [22])

\section{Research Result and Discussion}

a.Determining the value of eccentricity and comparing it with Actual Value.

The eccentricity value is obtained from the maximum value of angular perigee diameter and minimum angular diameter apogee by using equation (9). To determine the angular value of perigee diameter and angular value of apogee diameter can be seen in Figure 6. The highest or maximum diameter angular value is the perigee diameter angular value and the lowest or minimum value is the angular value of apogee diameter.

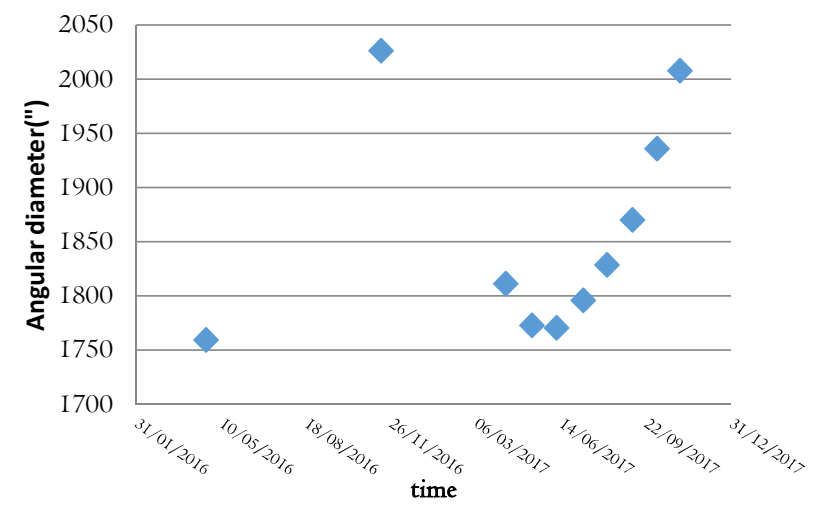

Figure 6. Graphical the relation of angular diameter to time from the results of the Image Analysis Technique

After obtaining the eccentricity value experimentally, it can be concluded that the eccentricity value experimentally and the actual value of $22 \%$ can be seen in table $\mathrm{I}$. The relatively larger error due to the actual value obtained from the observation results hundreds of years or even thousands of years is then averaged so that the actual value is obtained. The value of error is quite large due to data collection done up to IO months because there are several obstacles. The constraints that often occur are weather. The weather greatly affects the quality of shooting the full moon and the full moon is less than once in a span of 27 or 28 days. In addition, the telescope used also has drawbacks. The telescope used is a refractor telescope or a lens telescope that will affect the image of the Full Moon image received.
Measurement Eccentricity the Moon's Orbit with Image Analysis...

Table I. Relative errors between experiment and Actual Value

\begin{tabular}{ccc}
\hline Eksperimen & Actual Value & Ralat \\
\hline 0,07052779 & 0,0549 & $22 \%$ \\
\hline
\end{tabular}

Based on the experimental calculations the sexentricity values obtained were $0.07 \pm 0.01$. The existence of differences in eccentricity values can be seen from NASA calculations over 5000 years of eccentricity values included in the susceptible value of 0.0255 to 0.0775 . While averaged over 5000 years the eccentricity value is 0.0549 [23].

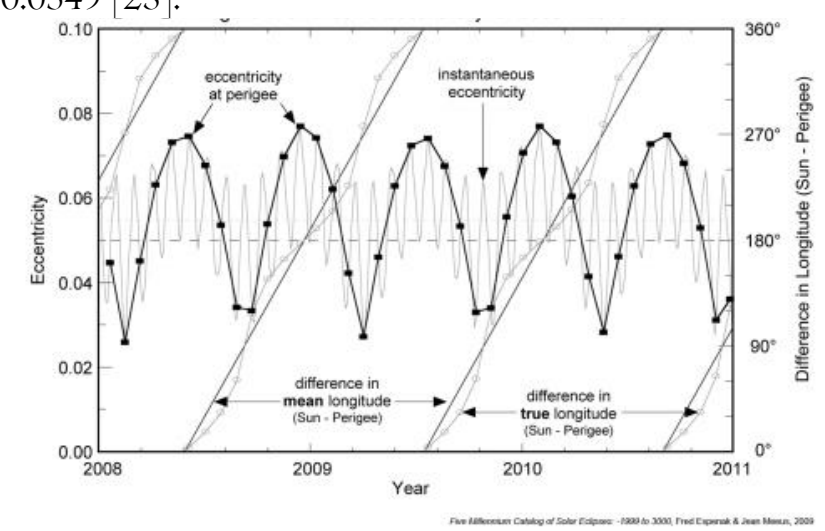

Figure 7. The moon eccentricity from 2008-2010 [23].

Figure 7 shows that eccentricity reaches the maximum when the main axis of the moon's orbit points directly towards or directly away from the Sun $\left(0^{\circ}\right.$ and $\left.180^{\circ}\right)$. This occurs at an average interval of 205.9 days, which is longer than half a year because of the shift of the main axis to the east. Eccentricity reaches a minimum point when the main axis of the Moon's orbit is perpendicular to the Sun $\left(90^{\circ}\right.$ and $\left.270^{\circ}\right)$ [23].

\section{b. Determining the Value of Eccentricity and Comparing with Ephemeris Value}

Ephemeris value is an accurate calculation that can calculate the angular diameter of the Moon from year to year so that it can be used as a reference value [24].

In Table $\mathrm{IO}$ is the difference of the Angular Diameter value Experimently and Emphemeris Values so that obtained the average value of error is $0.653 \%$. 
Table 2. The differences of Angular Diameter Values Experimentally and Emphemeris Values

\begin{tabular}{|c|c|c|c|}
\hline Date & $\begin{array}{l}\text { Angular Diameter } \\
\text { Value } \\
\text { Exsperimentally }\end{array}$ & $\begin{array}{l}\text { Angular Diameter } \\
\text { Value } \\
\text { Emphemeris }\end{array}$ & Deviation \\
\hline $22 / 04 / 2016$ & I759.179I28 & 1764 & $0.273 \%$ \\
\hline I4/II/20I6 & 2026.I5002I & 2009.2 & $0.844 \%$ \\
\hline I0/04/2017 & I8I0.84386 & I8I4.6 & $0.207 \%$ \\
\hline $\mathrm{II} / 05 / 2017$ & I772.639268 & 1769.3 & $0.189 \%$ \\
\hline $09 / 06 / 2017$ & 1770.155642 & I763.6 & $0.372 \%$ \\
\hline $10 / 07 / 2017$ & 1795.741158 & 1788.5 & $0.405 \%$ \\
\hline $07 / 08 / 2017$ & 1828.457749 & I 804.4 & $1.333 \%$ \\
\hline $06 / 09 / 2017$ & I869.7I4657 & I859.I & $0.571 \%$ \\
\hline $05 / 10 / 2017$ & I935.562327 & 1903.3 & $1.695 \%$ \\
\hline $0 \mathrm{I} / \mathrm{II} / 20 \mathrm{I7}$ & 2007.655893 & 1994.8 & $0.644 \%$ \\
\hline
\end{tabular}

From Table 8 can be described in the form of diameter angular graphs to the time with experiment values and emphemeris values in Figure 8.

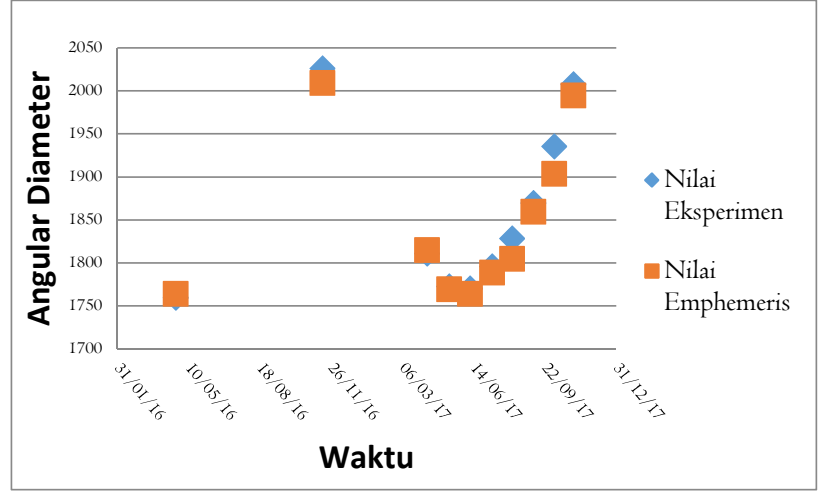

Figure 8. the graph of angular diameter relation to the time with Experimental and Emphemeris value data.

The eccentricity value which obtained from emphemeris measurements is 0.065 . So that the eccentricity value is measured by the experimental value and emphemeris value, the errors obtained by $8 \%$ can be seen in Table 3 .

Table 3. Relative error between experiment and ephemeris

\begin{tabular}{ccc}
\hline Eksperimen & Emphemeris & Ralat \\
\hline 0,07052779 & 0,06509754 & $8 \%$ \\
\hline
\end{tabular}

\section{Conclusion}

Based on the results of the research and discussion, it was concluded that the measuring accuracy of the Moon disk diameter using the Tracker can be viewed from the eccentricity value obtained.
The eccentricity value obtained has an error of $22 \%$ if compared to the actual value, whereas if the eccentricity value obtained compared to the ephemeris value, the error value is $8 \%$. The eccentricity value obtained was $0.07 \pm$ 0.0I. This is in accordance with first Kepler's first law which states that the orbit of each planet and satellite are ellipses and has an eccentricity value of $0<\mathrm{e}<\mathrm{I}$.

\section{Acknowledgment}

Thank you to PASTRON and ANDROMEDAthey helpedto guide the continuity of this experimental research and thank you to all friends who helped us in conducting this experiment.

\section{Bibliography}

[I] Subagya, Hari, and InsihWilujeng. Fisika SMA/MA Kelas $X$ Kurikulum 2013 KelompokPeminatan MIPA [Physics for high school]. Jakarta: BUMI AKSARA, 2013.

[2] Sutantyo, Winardi. PengantarAstrofisikaBintangBintang di AlamSemesta [Introduction to astrophysics of stars in the space]. Bandung: ITB, 2010.

[3] Kunjaya, Chatief. SuplemenAstronomiuntuk SMA[Supplement of astronomy for high school]. Bandung: PT TrisulaAdisakti, 2014.

[4] Dutton, John A. Astro 80I. 2017. https://www.e-ducation.psu.edu/astro80I / content/12_p4.html (accessed June 7, 2017).

[5] Osler. J, Thomas. An unusual approach to Kepler's first law.American Association of Physics Teachers, 200I: 69.

[6] Roy, A E, and D Clarke. AstronomyPrinciple andPractice. Bristol: Institute of Physics Publishing, 2012.

[7] Morison, Ion. Introduction to Astronomy and Cosmolgy. University of Manchester, UK: Wiley, 2008.

[8] Sarton, E Jay. Measuring the moon's orbit. The Physics Teacher, 1980: 504-508.

[9] Williams, David. NASA. July 3, 2017. https://nssdc.gsfc.nasa.gov/planetary/factsheet/ moonfact.html (accessed July I5, 2017).

[IO] Oostra, Benjamin. Measuring the Moon's orbit using a hand-held camera.American Association of Physics Teachers, 2014: 317-32I.

[II] Zuluaga, Jorge I., Juan C. Figueroa, and Ignacio Ferrin. The Simplest method the Geocentric lunar Distance: a case of citizen science.American Journal of Physics, 20I4: I-I6. 
Tanzilla et al.

[12] Oostra, Benjamin. Introducing the Moon's Orbital Eccentricity.American Association of Physics Teacher, 2014: 460-462.

[13] Krisciunas, Kevin. Determning the eccentricity of the Moon's Orbit without a Telescope.American Association of Physics Teachers, 2010: 834-838.

[I4] Tanzilla, Ricka, Lukman Hakim, and YudhiaktoPramudya. Pengukur Diameter TampakBulanpadaPosisi Perigee dan Apogee [Measure of Moon apparent Diameter at the Position of Perigee and Apogee].Seminar Quantum 2016. Yogyakarta: Program StudiPendidikanFisika, Universitas Ahmad Dahlan, 2016. 27 I-274.

[15] Angelo, Joseph A. Encyclopedia of Space and Astronomy. New York: Library of Congress Cataloging, 2006.

[16] Wilson, Robert. Astronomy throughThe Ages. Francis: Taylor \& Francis e-Library, 2005.

[17] Kindersley, Dorling. JendelaOptik Astronomy [Optic window of astronomy]. Jakarta: PT BalaiPustaka, 2007.
Indonesian Review of Physics, Volume I, Number I, 2018 Measurement Eccentricity the Moon's Orbit with Image Analysis...

[I8] Dunford, Bill. Solar System. July 5, 2016. https://solarsystem.nasa.gov/planets/moon/inde pth (accessed July 2I, 20I7).

[19] Yani, Ahmad. PengantarKosmografi [Introduction of Cosmography]. Yogyakarta: PenerbitOmbak, 2014.

[20] Denevi, Brett. The Moon.Physics Today, 2017: $39-43$.

[2I] Celletti, Alessandra. Stability and Chaos in Celestial Mechanics. UK: Chichester, 2010.

[22] Seeds, Micheal A. The Solar System, Sixth Edition. USA: THOMSON, 2008.

[23] Young, Alex. Eclipse and The Moon's Orbit. January I2, 20I2. https://eclipse .gsfc.nasa.gov/SEhelp/moonorbit.html (accessed December 29, 2017).

[24] Espenak, Fred. AstroPixcel.January I3, 2015. http://astropixels.com/ephemeris/moon/moon2 017.html (accessed December 30, 2017). 